\title{
Brain-derived neurotrophic factor levels and bipolar disorder in patients in their first depressive episode: 3-year prospective longitudinal study
}

Zezhi Li,* Chen Zhang, * Jinbo Fan, Chengmei Yuan, Jia Huang, Jun Chen, Zhenghui Yi, Zuowei Wang, Wu Hong, Yong Wang, Weihong Lu, Yangtai Guan, Zhiguo Wu, Yousong Su, Lan Cao, Yingyan Hu, Yong Hao, Mingyuan Liu, Shunying Yu, Donghong Cui, Lin Xu, Yanyan Song and Yiru Fang

\section{Background}

Early identification of patients with bipolar disorder during their first depressive episode is beneficial to the outcome of the disorder and treatment, but traditionally this has been a great challenge to clinicians. Recently, brain-derived neurotrophic factor (BDNF) has been suggested to be involved in the pathophysiology of bipolar disorder and major depressive disorder (MDD), but it is not clear whether BDNF levels can be used to predict bipolar disorder among patients in their first major depressive episode.

\section{Aims}

To explore whether BDNF levels can differentiate between MDD and bipolar disorder in the first depressive episode.

\section{Method}

A total of 203 patients with a first major depressive episode as well as 167 healthy controls were recruited. After 3 years of bi-annual follow-up, 164 patients with a major depressive episode completed the study, and of these, 21 were identified as having bipolar disorder and 143 patients were diagnosed as having MDD. BDNF gene expression and plasma levels at baseline were compared among the bipolar disorder, MDD and healthy control groups. Logistic regression and decision tree methods were applied to determine the best model for predicting bipolar disorder at the first depressive episode.

\section{Results}

At baseline, patients in the bipolar disorder and MDD groups showed lower BDNF mRNA levels $(P<0.001$ and $P=0.02$ respectively) and plasma levels $(P=0.002$ and $P=0.01$ respectively) compared with healthy controls. Similarly, BDNF levels in the bipolar disorder group were lower than those in the MDD group. These results showed that the best model for predicting bipolar disorder during a first depressive episode was a combination of BDNF mRNA levels with plasma BDNF levels (receiver operating characteristics $(\mathrm{ROC})=0.80$, logistic regression; $\mathrm{ROC}=0.84$, decision tree) .

\section{Conclusions}

Our findings suggest that BDNF levels may serve as a potential differential diagnostic biomarker for bipolar disorder in a patient's first depressive episode.

\section{Declaration of interest}

None.
Bipolar disorder is a highly prevalent, recurrent and disabling mental illness worldwide, and is often accompanied by high mortality rates, comorbity and economic burdens resulting from suicide and other related medical illnesses. ${ }^{1,2}$ Although biological research on bipolar disorder has expanded significantly, there has been no precise understanding as to its pathophysiology. Furthermore, distinguishing between major depressive disorder (MDD) and bipolar disorder with depressive episodes remains a diagnostic challenge for clinicians, especially when a patient presents during their first major depressive episode.

Finding novel biomarkers for bipolar disorder, however, has been repeatedly considered to be a crucial breakthrough in combating these challenges, so much so that the development of a reliable and robust diagnostic test has been thought of as the longstanding 'Holy Grail' in psychiatry. ${ }^{3}$ Finding novel biomarkers is critical to understanding the underlying pathophysiology of bipolar disorder and developing effective psychopharmacological treatment. Furthermore, finding these biomarkers capable of identifying patients with bipolar disorder early in their first depressive episode would allow for early intervention and thereby improve the outcome of the disorder and therapeutic treatments.

*These authors contributed equally to this work.
In the search for potential bipolar disorder biomarkers, an increasing number of studies strongly suggest that a neurobiological basis may underlie the aetiology of bipolar disorder. In particular, brain-derived neurotrophic factor (BDNF) has attracted attention for its key role in mediating neuronal survival, growth, plasticity and connectivity as well as synaptic efficacy, all of which are thought to be involved in the pathophysiology of bipolar disorder. $^{4,5}$ Growing evidence has shown that patients with bipolar disorder displayed lower BDNF levels in both peripheral and central nervous system tissues compared with healthy controls. ${ }^{6,7}$ Moreover, a meta-analysis demonstrated that BDNF levels decreased in patients with bipolar disorder during manic or depressive phases, ${ }^{8}$ and another study noted that moodstabilising drugs such as lithium and valproate - the most commonly used treatment options for bipolar disorder - were previously shown to increase BDNF levels in hippocampal tissues. $^{9,10}$ Interestingly, however, down-regulated BDNF levels were also reported in patients with MDD. ${ }^{11-13}$ It remains to be seen whether or not BDNF levels can differentiate between MDD and bipolar disorder in the first depressive episode or whether BDNF levels can predict bipolar disorder in patients with a first major depressive episode.

In the present study, patients who were drug-naive and in their first major depressive episode were recruited and followed 
for 3 years to identify which individuals were diagnosed with bipolar disorder and MDD. Presuming that BDNF mRNA and/ or plasma BDNF levels on admission could differentiate two patient groups (patients with either MDD or bipolar disorder in their first depressive episode), we re-examined blood samples taken from the patients before they were diagnosed with either bipolar disorder or MDD, to see whether or not BDNF levels provided any clues for establishing a reliable predictor.

\section{Method}

\section{Participants}

Participants were recruited from the Shanghai Mental Health Center between January 2007 and January 2009. The parameters and methodologies of the study were reviewed and approved by the Institutional Review Board of Shanghai Mental Health Center, and all protocols related to human experiments were conducted in accordance with the Declaration of Helsinki. We ensured that all participants were given an adequate understanding of the study and written informed consent was obtained from all individuals prior to their inclusion in the study.

All participants underwent the Structured Clinical Interview for DSM-IV-TR Axis I Disorders-Patient Edition (SCID-I/P), ${ }^{14}$ following which, demographic data on age, gender, body mass index (BMI), the duration of major depressive episode, history of smoking, alcohol and other drug use was collected. Assessments of the Hamilton Rating Scale for Depression-17 (HRSD-17) ${ }^{15}$ and Young Mania Rating Scale (YMRS) ${ }^{16}$ were conducted independently by two experienced psychiatrists (interrater reliability, kappa $=0.84$ and kappa $=0.81$ respectively) on admission.

For the purposes of this study, patients with a first depressive episode, who were drug-naive (i.e. never taken any psychotropic medication), aged $19-50$ years old and who met DSM-IV ${ }^{17}$ criteria for a major depressive episode, with an HRSD-17 score of $\geqslant 17$, were recruited. Patients with other comorbid Axis I psychiatric disorders were excluded, including those with anxiety disorder, schizophrenia, nicotine dependence, alcohol dependence and substance dependence. Patients with a history or current use of alcohol, nicotine and other substances were also excluded (in this study, smoking and/or alcohol use refers to smoking and/or alcohol harmful use or misuse, excluding recreational use). Patients with severe medical illness (e.g. cancer, diabetes), organic brain disease and those who were pregnant were excluded to ensure clarity.

Age- and gender-matched healthy controls were recruited by advertisement, and those whose HRSD-17 score was $<7$ were enrolled. Individuals with any major Axis I disorder (including substance dependence, psychotic disorder, mood disorder and anxiety disorder), family history of mental disorder or severe physical diseases (e.g. hypertension, diabetes, cancer) were excluded. In total, 203 patients with a mean age of 31.1 years (s.d. $=4.8)(47$ males and 156 females) and 167 healthy controls with a mean age of 30.9 years (s.d. $=4.5) \quad(35$ males and 132 females) were included on admission.

\section{RNA and plasma preparation}

On admission, $20 \mathrm{ml}$ peripheral venous blood of fasting patients and healthy controls were collected between $07.00 \mathrm{~h}$ and $09.00 \mathrm{~h}$. Total RNA was extracted from $10 \mathrm{ml}$ peripheral blood samples using the QIAamp RNA blood Mini Kit (Qiagen, Chatsworth, California, USA) and then treated with DNase (Qiagen, Chatsworth, California, USA). The complementary DNA (cDNA) was synthesised by incubating DNase-treated total RNA $(1.0 \mu \mathrm{g})$ with omniscript reverse transcription reagents (Qiagen, Chatsworth, California, USA) and a random primer according to the manufacturer's protocols. Plasma samples were separated from $10 \mathrm{ml}$ peripheral venous blood and centrifuged at $3500 \mathrm{rpm}$ at $4^{\circ} \mathrm{C}$ for $20 \mathrm{~min}$. All plasma samples were then frozen to $-80^{\circ} \mathrm{C}$.

\section{Gene relative expression levels analysis by quantitative reverse transcription polymerase chain reaction (RT-PCR)}

BDNF mRNA expression levels were measured by quantitative RT-PCR using an ABI Prism 7900HT Sequence Detection System (Applied Biosystems, California, USA) with a 384-well format. For the RNA internal control, we used glyceraldehyde-3-phosphate dehydrogenase (GAPDH; Applied Biosystems, California, USA) mRNA expression to normalise the target gene expression levels. TaqMan Universal PCR MasterMix and TaqMan probes/primers were obtained from Applied Biosystems. Quantitative RT-PCR reaction was carried out as follow: $50^{\circ} \mathrm{C}$ for $2 \mathrm{~min}$ and $95^{\circ} \mathrm{C}$ for $10 \mathrm{~min}$, then $95^{\circ} \mathrm{C}$ for 50 cycles of $10 \mathrm{~s}, 59^{\circ} \mathrm{C}$ for $1 \mathrm{~min}$. Experiments were performed in triplicate for each sample.

Results of the real-time PCR data were represented as cycle threshold $(\mathrm{Ct})$ value, defined as the threshold cycle of PCR at which a significant increase in the fluorescence signal was first detected. Data were collected and analysed with Sequence Detector Software 2.1 (Applied Biosystems, California, USA). The comparative $\mathrm{Ct}$ value $(\Delta \mathrm{Ct})$ was used for relative expression in target gene product, and $2-\Delta \mathrm{Ct}$ represents the relative expression level. The $\Delta \mathrm{Ct}$ value of each sample (patients and controls) was obtained by subtracting the average GAPDH Ct value of each sample from the average target gene Ct value of each sample.

\section{Plasma levels analysis by enzyme-linked immunosorbent assay (ELISA)}

Each plasma sample was measured in duplicate by the ELISA method according to the manufacturer's protocols (R\&D Systems, Minneapolis, Minnesota, USA). Several sample measurements were repeated to confirm reproducibility of the assay and the inter-assay coefficient of variation was $4.18 \%$. Researchers were masked to the clinical data of all participants.

\section{Naturalistic follow-up}

During the naturalistic follow-up, patients could present to the out-patient department for treatment as needed. However, they were asked to be interviewed and assessed by two experienced psychiatrists from our research team every 6 months, using the SCID-I/P, YMRS and HRSD-17. Patients diagnosed with bipolar disorder or those who had a manic episode during the 3-year study period were identified as having bipolar disorder. The primary end-point was the occurrence of a hypomanic or manic episode.

\section{Data analysis and statistical tests}

Demographic data were analysed using chi-squared, $t$-test or ANOVA (one-way) as appropriate. Data were examined for normality using the Kolmogorov-Smirnov test. As the plasma BDNF levels were not normally distributed, they were transformed into normal distribution using natural logarithms prior to statistical analysis. ANOVA (one-way) followed by Bonferroni multiple comparison test was used to analyse the difference of BDNF expression levels or plasma levels among patients with MDD (MDD group), patients with bipolar disorder (BPD group) and healthy controls on admission. Because several previous studies demonstrated that age, gender and BMI might affect BDNF levels, ${ }^{18,19}$ linear regression models were used to analyse 
the correlation between BDNF levels and HRSD-17 in the MDD and BPD groups on admission. Confounding factors such as age, gender, illness duration and BMI were also evaluated in a linear regression model to balance their effects. To determine the best model for differentiating bipolar disorder from MDD at the first depressive episode, the discriminatory capacity of each model (gene expression level of BDNF, plasma BDNF level, and the combination thereof) was analysed by calculating the area under the receiver operating characteristic (ROC) curve using logistic regression and a decision tree. Decision trees are predictive models mapping observations about an item to a conclusion on its target value. ${ }^{20}$ Classification and regression (CRT) algorithms were used to build this classification model. In these tree structures, leaves represent classifications and branches represent conjunctions of features that cause those classifications. A tenfold cross-validation was applied to detect the efficiency of this technique. $^{21}$

A value of 0.5 indicated that the model is equivalent to pure chance, whereas a value of 1 indicated perfect discrimination; concordance statistics between 0.7 and 0.8 were generally considered acceptable. ${ }^{22}$ The optimal cut-off value was defined by ROC. Throughout all analyses, a level of 0.05 was assumed to be significant. Statistical analyses were conducted using SAS 9.2 for Windows (SAS Institute, Cary, North Carolina, USA).

\section{Results}

\section{Naturalistic follow-up}

A total of 203 patients with a major depressive episode were recruited into the study. After 3 years' follow-up, 39 patients dropped out of the study prior to the primary end-point as we were unable to contact them, leaving 164 patients that completed the study. Demographic data collected at the beginning of the study showed there was no difference between the 39 patients who dropped out and the 164 patients who completed the study in terms of age $(t=0.51, P=0.61)$, gender $\left(\chi^{2}=2.90, P=0.09\right)$, BMI $(t=0.86, P=0.39)$, the duration of the depressive episode $(t=0.48, \quad P=0.63)$ and HRSD-17 scores $(t=0.74, P=0.46)$. Among the 164 patients who completed the study, 21 patients were identified as having bipolar disorder (type I $n=6$, type II $n=15$ ) and 143 patients were diagnosed as having MDD (Fig. 1). Demographic data collected during the baseline visit from the two patient groups and the healthy controls (including age, gender, BMI, duration of depressive episode and HRSD-17 scores) were comparable, as shown in Table 1.

Relative expression levels of BDNF mRNA in the BPD group, MDD group and healthy controls on admission

We noted a significant difference in BDNF expression levels among the BPD group $(0.0064$, s.d. $=0.0023)$, the MDD group

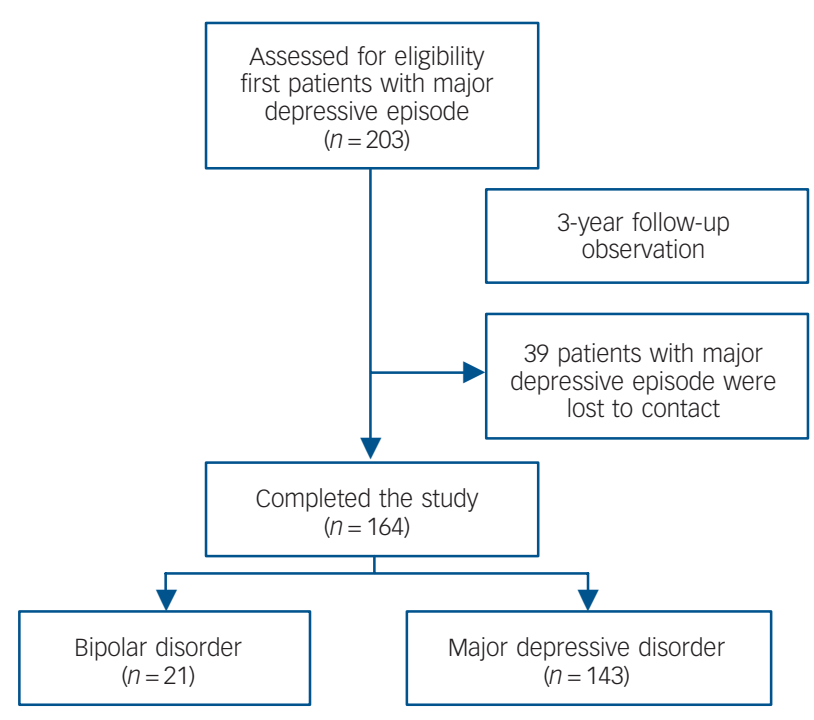

Fig. 1 Cohort diagram.

$(0.0081$, s.d. $=0.0022)$ and healthy controls $(0.0088$, s.d. $=0.0022)$ $(F=12.08$, d.f. $=2, P<0.001$ ). ANOVA (one-way) followed by Bonferroni multiple comparison test showed that BDNF levels were decreased in both the BPD group and the MDD group compared with the healthy controls $(P<0.001$ and $P=0.02$ respectively), but that $\mathrm{BDNF}$ levels in the $\mathrm{BPD}$ group were even lower than those in the MDD group $(P=0.004)$ (Fig. $2(\mathrm{a}))$. There was no observed difference in BDNF levels between patients with bipolar I and bipolar II disorder $(t=1.10$, d.f. $=19$, $P=0.28)$.

\section{Plasma BDNF levels in the BPD group, MDD group and healthy controls on admission}

There was a significant difference of plasma BDNF levels among the BPD group $(2.66$, s.d. $=0.50)$, the $\mathrm{MDD}$ group (2.86, s.d. $=0.41)$ and healthy controls $(3.00$, s.d. $=0.43)(F=8.35$, d.f. $=2, P<0.001$ ). ANOVA (one-way) followed by Bonferroni multiple comparison test showed that plasma BDNF levels were decreased in both the BPD and MDD groups compared with the healthy controls $(P=0.002$ and $P=0.01$ respectively), although no difference in plasma BDNF levels was found between the BPD and MDD groups $(P=0.16)$ (Fig. 2(b)). There was no observed difference in BDNF plasma levels between patients with bipolar I and bipolar II disorder $(t=0.91$, d.f. $=19$, $P=0.37)$.

\begin{tabular}{|c|c|c|c|c|c|}
\hline & $\begin{array}{l}\text { MDD group } \\
\quad(n=143)\end{array}$ & $\begin{array}{l}\text { BPD group } \\
\quad(n=21)\end{array}$ & $\begin{array}{l}\text { Healthy controls } \\
\qquad(n=167)\end{array}$ & $\begin{array}{l}t, F \\
\text { or } \chi^{2}\end{array}$ & $P$ \\
\hline Age, years: mean (s.d.) & $31.0(5.1)$ & $32.2(4.1)$ & $30.9(4.5)$ & 0.72 & 0.49 \\
\hline Male, $n(\%)$ & $36(25.2)$ & $6(28.6)$ & $35(21.0)$ & 1.12 & 0.57 \\
\hline Body mass index, mean (s.d.) & $21.7(2.3)$ & $21.4(3.5)$ & $21.6(2.6)$ & 0.16 & 0.85 \\
\hline Duration of depressive episode, months: mean (s.d.) & $2.8(1.0)$ & $2.8(0.8)$ & - & 0.06 & 0.95 \\
\hline Family history of mood disorder, $\mathrm{n}(\%)$ & $10(7.0)$ & $2(9.5)$ & - & 0.17 & 0.68 \\
\hline HRSD-17 score on admission, mean (s.d.) & $22.4(1.6)$ & $22.2(1.7)$ & - & 0.43 & 0.67 \\
\hline
\end{tabular}




\section{Association between BDNF expression/plasma levels and severity of disease}

To test the potential association between BDNF expression/plasma levels and the severity of disease on admission, linear regression models were used to clarify the potential effects of confounding factors. These models were composed of both independent (e.g. relative expression levels of BDNF or log-transformed plasma BDNF levels, age, gender, BMI and duration of depressive episode and dependent variables (HRSD-17 score). The results showed that neither the relative levels of BDNF or plasma BDNF levels were correlated with HRSD-17 in either the MDD or BPD group $(P>0.05)$ (Table 2).

\section{Best model to predict bipolar disorder in the first depressive episode}

Although there was no observed correlation between BDNF gene expression levels and plasma levels in the BPD group $(r=0.20$, $P=0.38$ ), there was a marginal correlation between BDNF gene expression level and plasma level in the MDD group $(r=0.21$, $P=0.02$ ). To determine the best model for predicting bipolar disorder in the first depressive episode, discriminatory capacity was analysed by calculating the area under the ROC curve using two different statistical methods: logistic regression and a decision tree. With logistic regression, the areas under the ROC curves of gene expression level of BDNF, plasma BDNF level and the combination of both were $0.69,0.61$ and 0.80 respectively (Fig. $3)$. Using a decision tree, the area under the ROC curve of the combination of BDNF expression levels with plasma BDNF levels was 0.84 (the process of a decision tree is shown in online Fig. DS1). Both these methods showed that BDNF expression

(a)

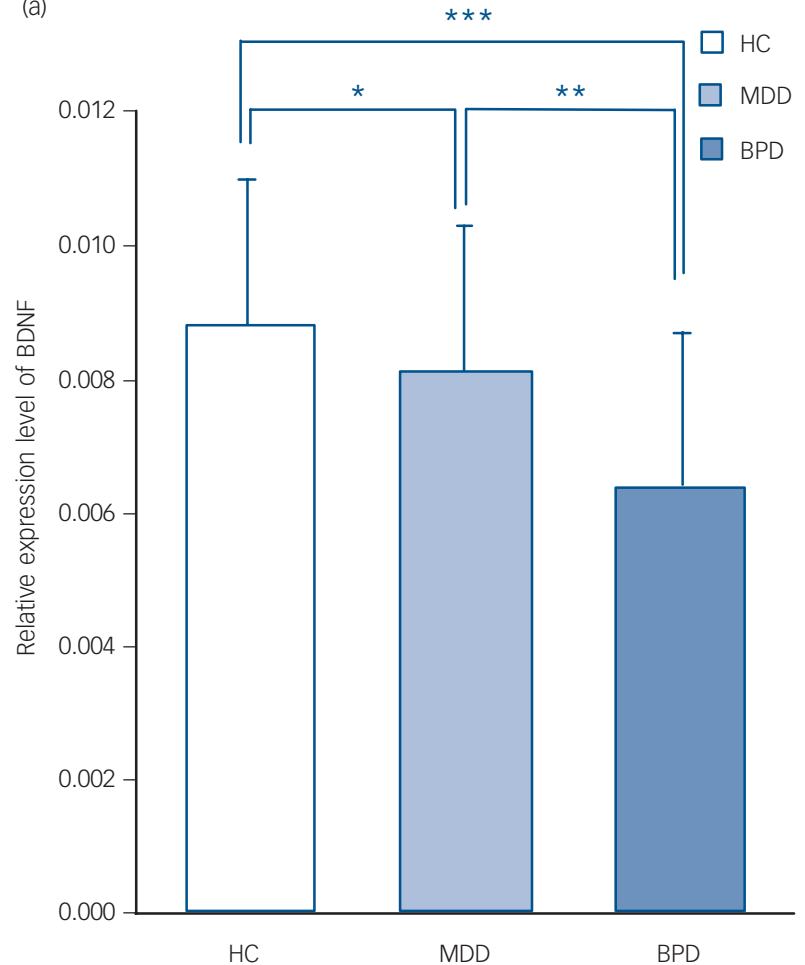

levels combined with plasma BDNF levels served as the most accurate model for predicting the occurrence of bipolar disorder in patients with a first major depressive episode.

We also conducted a sensitivity analysis, including the patients who dropped out. If we categorise these patients as having MDD, with logistic regression the areas under the ROC curves of gene expression level of BDNF, plasma BDNF level and the combination of both were $0.67,0.61$ and 0.73 respectively. Using a decision tree, the area under the ROC curve of the combination of BDNF expression levels with plasma BDNF levels was 0.84. In summary, missing values - whether deleted or identified as MDD - did not change the diagnosis value of the variables.

\section{Discussion}

Despite numerous advances in understanding the aetiology and underlying mechanisms of bipolar disorder, the outcome for many patients remains poor. Since improvements could be made with earlier intervention and treatment, there is a strong need to develop adequate early detection methods (e.g. BDNF biomarkers $)^{23}$ However, core clinical features overlap between bipolar disorder depression and MDD, and a depressive episode is often the first mood syndrome at the onset of bipolar disorder in particular. Finding a reliable and robust biomarker identifying bipolar disorder early during a patient's first presenting episode of depression is critical.

BDNF plays a critical role in neuronal processes including neurogenesis, neuronal survival, growth, plasticity and synaptic efficacy. Previous data have also indicated that BDNF-related neuronal function may be a crucial mediator of the effects of brain volume, psychosocial stress and psychopathology in mood

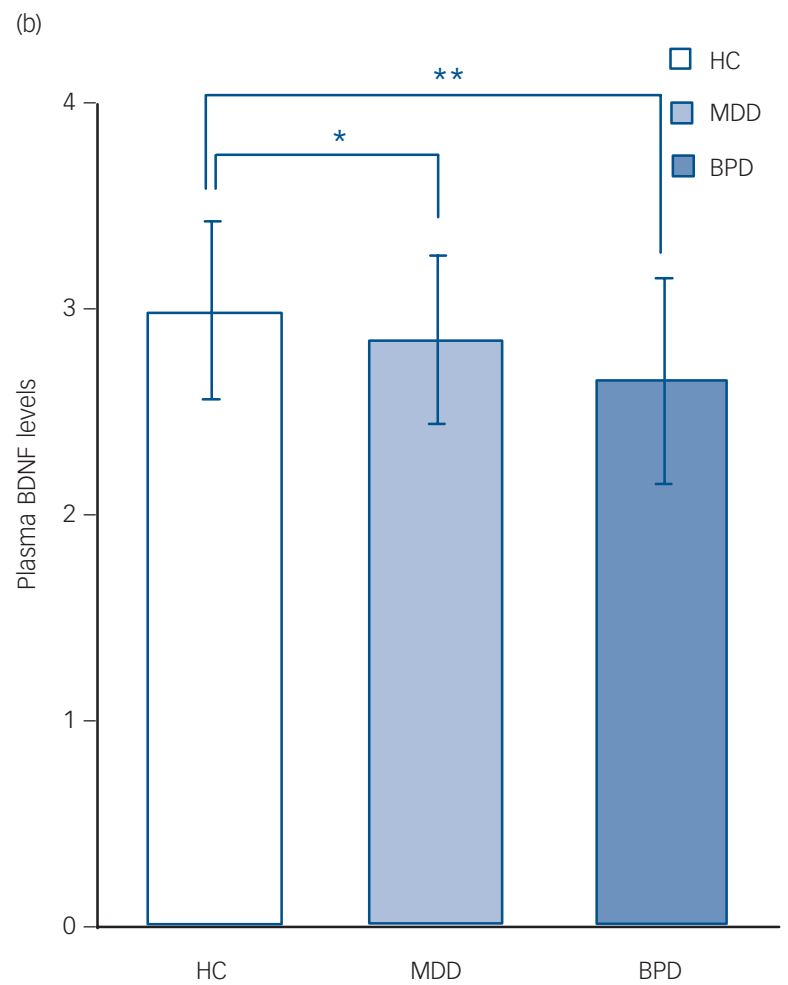

Fig. 2 Brain-derived neurotrophic factor (BDNF) levels in the three groups on admission (data presented as means (s.e.m.).

On admission, BDNF expression levels decreased in both the bipolar disorder (BPD) and major depressive disorder (MDD) groups compared with healthy controls (HCS); and BDNF expression levels in the BPD group were lower than those in the MDD group (ANOVA followed by Bonfrerroni multiple comparison test) (a). On admission, plasma BDNF levels decreased in the BPD and MDD groups compared with the HC group; but no difference of plasma BDNF levels was found between the BPD group and MDD group (ANOVA followed by Bonfrerroni multiple comparison test) (b). ${ }^{*} P<0.05 ;{ }^{*} P<0.005 ;{ }^{* \star \star} P<0.001$. 
Table 2 Linear regression models for association study ${ }^{a}$

\begin{tabular}{|c|c|c|c|c|c|c|c|c|c|c|c|c|}
\hline & \multicolumn{6}{|c|}{ MDD group } & \multicolumn{6}{|c|}{ BPD group } \\
\hline & \multicolumn{3}{|c|}{ BDNF expression levels } & \multicolumn{3}{|c|}{ Plasma BDNF levels ${ }^{b}$} & \multicolumn{3}{|c|}{ BDNF expression levels } & \multicolumn{3}{|c|}{ Plasma BDNF levels ${ }^{b}$} \\
\hline & $B$ & $t$ & $P$ & $B$ & $t$ & $P$ & $B$ & $t$ & $P$ & $B$ & $t$ & $P$ \\
\hline Age & 0.07 & 0.79 & 0.43 & 0.02 & 0.23 & 0.82 & -0.04 & -0.16 & 0.87 & -0.08 & -0.33 & 0.75 \\
\hline Gender & -0.12 & -1.33 & 0.19 & -0.12 & -1.38 & 0.17 & -0.17 & -0.63 & 0.54 & -0.07 & -0.25 & 0.81 \\
\hline Body mass index & 0.09 & 1.13 & 0.26 & 0.09 & 1.09 & 0.28 & 0.16 & 0.67 & 0.51 & 0.13 & 0.51 & 0.62 \\
\hline $\begin{array}{l}\text { Duration of } \\
\text { depressive episode }\end{array}$ & -0.10 & -1.18 & 0.24 & -0.04 & -0.48 & 0.63 & 0.15 & 0.59 & 0.57 & 0.19 & 0.70 & 0.50 \\
\hline HRSD-17 score & -0.11 & -1.25 & 0.22 & -0.07 & -0.78 & 0.44 & -0.39 & -1.56 & 0.14 & -0.31 & -1.18 & 0.26 \\
\hline
\end{tabular}

disorders. ${ }^{24}$ To the best of our knowledge, this is the first prospective longitudinal study to investigate whether BDNF expression levels and/or plasma levels are capable of predicting bipolar disorder during a first depressive episode. We performed a large-scale study in a cohort of patients with a first major depressive episode who were drug-naive, and followed them for 3 years to identify those with bipolar disorder. The low proportion of males in our sample was consistent with a recent systematic review about the epidemiology of major depressive disorder in mainland China. ${ }^{25}$ Our main results showed that gene expression levels of BDNF and plasma BDNF levels were both decreased in the BPD and MDD groups prior to treatment compared with the levels in healthy controls. Similarly, our analyses showed that a combination of gene expression levels of BDNF and plasma BDNF levels were the best models to predict bipolar disorder in patients with a first major depressive episode.

Despite promising results in our study, earlier reports show that BDNF levels among individuals with mood disorders is far from consistent. Some studies found decreased BDNF levels in the depressive phase of bipolar disorder, whereas others did not. De Oliveira's group, for example, found that serum BDNF levels among patients in the depressed phase of bipolar disorder were decreased compared with controls. ${ }^{26}$ Fernandes et al ${ }^{27}$ also found that patients in the depressed phase of bipolar disorder

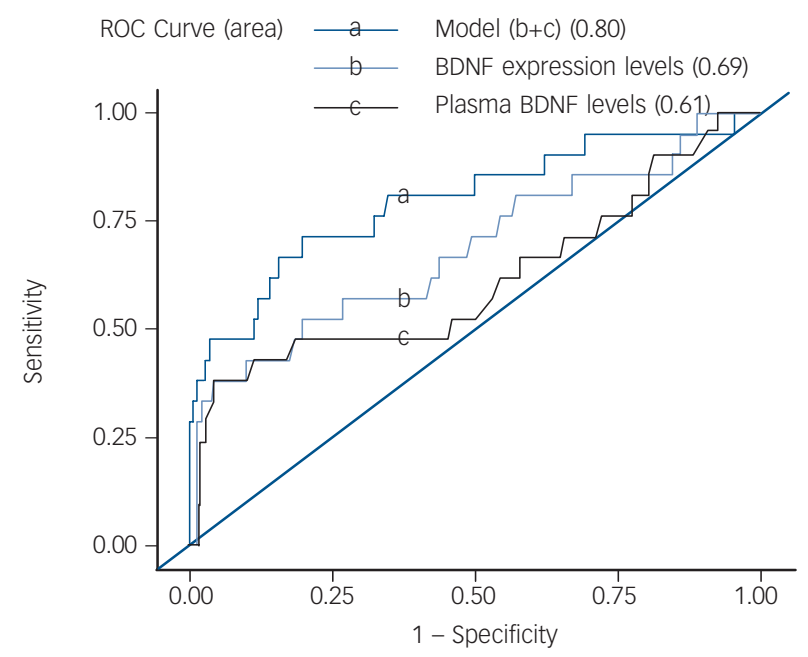

Fig. 3 Best model to predict bipolar disorder in first depressive episode.

Brain-derived neurotrophic factor (BDNF) expression levels combined with plasma BDNF levels was the best model to predict the occurrence of bipolar disorder in patients with major depressive episode using logistic regression (area under curve $=0.80$ ) and decision tree (online Fig. DS1). ROC, receiver operating characteristic. showed decreased BDNF levels compared with healthy controls. Machado-Vieira et $a l^{28}$ demonstrated that BDNF levels were significantly decreased in unmedicated patients with bipolar disorder during manic episodes compared with healthy controls and the severity of the manic episode was negatively correlated with plasma BDNF levels. However, Mackin et al ${ }^{29}$ found that BDNF levels in patients during the depressed phase of bipolar disorder were similar to those in healthy controls. One metaanalysis of 107 patients and 118 healthy participants demonstrated that BDNF levels decreased during a bipolar disorder depressive episode compared with healthy controls. ${ }^{8}$ Such inconsistent results are likely due to the comparatively small sample sizes used as well as the unspecified (or undetermined) psychopharmacological states of the patients (i.e. drug-naive $v$. medicated patients, or distinct medications being given).

To the best of our knowledge, no study to date has examined BDNF levels in patients with bipolar disorder in their first depressive episode. Similarly, our larger sample size (composed of patients who were drug-naive and in a first major depressive episode) provides provocative evidence that, compared with healthy controls, both BDNF expression levels and plasma levels have already decreased in patients with bipolar disorder even during their first depressive episode. Our results also showed that BDNF levels (both expression levels of BDNF and plasma levels) were lower in the MDD group than in healthy controls, consistent with most previous studies. ${ }^{30}$ Further evidence from several magnetic resonance imaging (MRI) studies has shown a decreased expression of BDNF in volumetric brain reductions in psychiatric disorders. ${ }^{31,32}$ Arnone et $a l^{33}$ previously reported that experiencing depression is associated with a decrease in hippocampal volume and an increase in grey matter following clinical improvement. Our findings, as well as those previously reported, strengthen the hypothesis that down-regulated BDNF levels, although playing a vital role in a number of developmental processes, synapticplasticity and reconstruction, ${ }^{13}$ may also be involved in the pathophysiology of both MDD and bipolar disorder.

Previous reports suggested that BDNF is a physiopathological biomarker in psychiatry. ${ }^{30,34,35}$ However, even though BDNF can be considered a biomarker, whether BDNF levels can predict bipolar disorder in a first depressive episode is not clear. Fernandes et $a l^{27}$ first reported that serum BDNF levels can differentiate MDD and bipolar disorder and that serum BDNF levels were lower in patients with bipolar disorder than those in either patients with MDD or healthy controls. Their observations, however, were based on a study of 10 patients with MDD, 40 patients with bipolar disorder and 30 healthy controls. In their cross-sectional study, patients with MDD and bipolar disorder were diagnosed at baseline, thus serum BDNF levels could not be used to predict bipolar disorder in patients in their first 
depressive episode. Furthermore, in the absence of a follow-up observation, it is not possible to rule out hypomanic or manic episodes in the future. More importantly, their samples were medicated patients, which could confound the results, a problem shared in most studies, ${ }^{36}$ because both mood stabilisers and antidepressants may potentially affect BDNF levels. ${ }^{37,38}$ They also performed a meta-analysis, which still found peripheral BDNF as a state-marker of mood episodes in bipolar disorder. ${ }^{8}$

In the present study, we found that gene expression levels of BDNF were lower in patients with bipolar disorder initially presenting a depressive episode compared with patients with MDD. The most effective model for predicting bipolar disorder in a first depressive episode was a combination of BDNF gene expression and plasma BDNF levels. These results suggest that BDNF level may be a potential biomarker for bipolar disorder that can be detected in the first depressive episode.

Interestingly, our results showed that the expression level of BDNF was lower in the BPD group than in the MDD group. Previously, Arnone et $a l^{39,40}$ and Kempton et $a l^{41}$ conducted meta-analysis studies which indicated that volumetric brain reductions in depression appeared localised in specific brain regions; conversely, there may be a more widespread volumetric loss in bipolar disorder that results in decreased intracranial brain volume. If true, this may, at least to some degree, explain the lower BDNF levels in bipolar disorder and reflect a different magnitude of genetic expression. This is only speculation, but it is an intriguing possibility that warrants further study.

\section{Limitations}

Despite the suggestive results, there are some limitations to consider. First, although we followed patients with a first major depressive episode for 3 years in an attempt to identify those patients with bipolar disorder, some patients may have experienced manic or hypomanic episodes in the following years. A longer longitudinal follow-up is required in the future to further confirm or clarify our results. Second, we performed a naturalistic observation, and patients were interviewed by our research team every 6 months, so YMRS scores were not obtained when manic/hypomanic symptoms actually occurred. Finally, most patients were at remission stage when they were interviewed and some patients were interviewed at their house every 6 months, so most of them did not want to have their blood drawn and therefore their BDNF levels were not obtained.

\section{Implications}

This study represents the first prospective longitudinal attempt to explore a predictive biomarker for bipolar disorder among patients in their first depressive episode. Our findings demonstrate that a combination of gene expression levels of BDNF and plasma BDNF levels may potentially serve to predict bipolar disorder in those experiencing their first depressive episode on admission; and it could potentially be significant in understanding the biological discrimination of affective disorders. Ultimately, down-regulated BDNF may contribute to the pathophysiology of bipolar disorder and MDD, although further evidence is needed to make any definitive determination.

\section{Funding}

This study was supported by the National Natural Science Foundation of China ( 91232719 30971047, 81000581, 81171272), National High-tech R\&D Program (863 Program 2006AA02Z430, Ministry of Science and Technology of China), the "12th Five-year Plan" of National Key Technologies R\&D Program (2012BAI01B04, Ministry of Science and of National Key Technologies R\&D Program (2012BAl01B04, Ministry of Science and
Technology of China), National Key Clinical Disciplines at Shanghai Mental Health Center (OMA-MH, 2011-873), Shanghai Natural Science Foundation (13ZR1460500, 10ZR1426600),
Shanghai Health Bureau Project, Shanghai Changhai Hospital Foundation, and Postdoctoral Grant of Secondary Military Medical University.

\section{Acknowledgements}

The authors are very grateful to all participants. We especially express our gratitude to Mrs Mary Beth Serrano from Case Western Reserve University, USA, for grammatical assistance.

Zezhi Li, MD, PhD, Division of Mood Disorders, Shanghai Mental Health Center Shanghai Jiao Tong University School of Medicine, Shanghai, and Department of Neurology, Shanghai Changhai Hospital, Secondary Military Medical University, Shanghai, China; Chen Zhang, MD, PhD, Division of Mood Disorders, Shanghai Mental Health Center, Shanghai Jiao Tong University School of Medicine, Shanghai, and Key Laboratory of Animal Models and Human Disease Mechanisms of the Chinese Academy of Sciences \& Yunnan Province, Kunming Institute of Zoology, Kunming, Yunnan, China; Jinbo Fan, PhD, Department of Epidemiology and Biostatistics, Case Western Reserve University School of Medicine, USA; Chengmei Yuan, MD, PhD, Jia Huang, MD, Jun Chen, MD, PhD, Zhenghui Yi, MD, PhD, Yuan, $M D$, PhD, Jia Huang, $M D$, Jun Chen, MD, PhD, Zhenghui Yi, MD, PhD,
Zuowei Wang, MD, PhD, wu Hong, MD, PhD, Yong Wang, MD, PhD, Weihong Lu, $\mathrm{MD}$, PhD, Division of Mood Disorders, Shanghai Mental Health Center, Shanghai Jiao Tong University School of Medicine, Shanghai, China; Yangtai Guan, MD, PhD, Department of Neurology, Shanghai Changhai Hospital, Secondary Military Medical University, Shanghai, China; Zhiguo Wu, MD, PhD, Yousong Su, MD, Lan Cao, MD, Yingyan Hu, MD, Division of Mood Disorders, Shanghai Mental Health Center Shanghai Jiao Tong University School of Medicine, Shanghai, China; Yong Hao, MD, PhD, Mingyuan Liu, MD, PhD, Department of Neurology, Shanghai Changhai Hospital, Secondary Military Medical University, Shanghai, China; Shunying Yu, MD, PhD, Donghong Cui, MD, PhD, Department of Genetics, Shanghai Mental Health Center, Shanghai Jiao Tong University School of Medicine, Shanghai, China; Lin Xu PhD, Key Laboratory of Animal Models and Human Disease Mechanisms of the Chinese Academy of Sciences \& Yunnan Province, Kunming, Yunnan, China: Yanyan Song, PhD, Department of Pharmacology and Biostatistics, Institute of Medical Sciences, Shanghai Jiaotong University School of Medicine, Shanghai, China; Yiru Fang, MD, PhD, Division of Mood Disorders, Shanghai Mental Health Center, Shanghai Jiao Tong University School of Medicine, Shanghai, China

Correspondence: Dr Yiru Fang, Division of Mood Disorders, Shanghai Mental Health Center, Department of Psychiatry, Shanghai Jiao Tong University School of Medicine, 600 South Wan Ping Road Shanghai 200030, China. Email: yirufang@gmail.com. Dr Yanyan Song (statistical analysis), Department of Biostaistics, Institute of Medical Sciences, Shanghai Jiaotong University School of Medicine, Shanghai, China. Email: yanyansong@sjtu.edu.cn

First received 19 Jun 2013, final revision 26 Dec 2013, accepted 27 Jan 2014

\section{References}

1 Dutta R, Boydell J, Kennedy N, van Os J, Fearon P, Murray RM. Suicide and other causes of mortality in bipolar disorder: a longitudinal study. Psychol Med 2007; 37: 839-47.

2 Lloyd T, Kennedy N, Fearon P, Kirkbride J, Mallett R, Leff J, et al. Incidence of bipolar affective disorder in three UK cities: results from the AESOP study. Br J Psychiatry 2005; 186: 126-31.

3 Le-Niculescu H, Kurian SM, Yehyawi N, Dike C, Patel SD, Edenberg HJ, et al. Identifying blood biomarkers for mood disorders using convergent functional genomics. Mol Psychiatry 2009; 14: 156-74.

4 Duman RS, Malberg J, Nakagawa S, D'Sa C. Neuronal plasticity and survival in mood disorders. Biol Psychiatry 2000; 48: 732-9.

5 Einat $\mathrm{H}$, Manji HK. Cellular plasticity cascades: genes-to-behavior pathways in animal models of bipolar disorder. Biol Psychiatry 2006; 59: 1160-71.

6 Dunham JS, Deakin JF, Miyajima F, Payton A, Toro CT. Expression of hippocampal brain-derived neurotrophic factor and its receptors in Stanley consortium brains. J Psychiatr Res 2009; 43: 1175-84.

7 Rajkowska G. Postmortem studies in mood disorders indicate altered numbers of neurons and glial cells. Biol Psychiatry 2000; 48: 766-77.

8 Fernandes BS, Gama CS, Ceresér KM, Yatham LN, Fries GR, Colpo G, et al. Brain-derived neurotrophic factor as a state-marker of mood episodes in bipolar disorders: A systematic review and meta-regression analysis. J Psychiatr Res 2011; 45: 995-1004.

9 Fukumoto T, Morinobu S, Okamoto Y, Kagaya A, Yamawaki S. Chronic lithium treatment increases the expression of brain-derived neurotrophic factor in the rat brain. Psychopharmacology (Berl) 2001; 158: 100-6.

10 Frey BN, Andreazza AC, Cereser KM, Martins MR, Valvassori SS, Réus GZ, et al. Effects of mood stabilizers on hippocampus BDNF levels in an animal model of mania. Life Sci 2006; 79: 281-6.

11 Brunoni AR, Lopes M, Fregni F. A systematic review and meta-analysis of clinical studies on major depression and BDNF levels: implications for the role of neuroplasticity in depression. Int J Neuropsychopharmacol 2008; 11: 1169-80. 
12 D'Sa C, Duman RS. Antidepressants and neuroplasticity. Bipolar Disord 2002; 4: 183-94.

13 Kelleher RJ, Govindarajan A, Tonegawa S. Translational regulatory mechanisms in persistent forms of synaptic plasticity. Neuron 2004; 44: 59-73.

14 First MB, Spitzer R, Gibbon M, Williams JBW. Structured Clinical Interview fo DSM-IV Axis I Disorders. Patient Edition (SCID-I/P. Version 2.0). Biometrics Research Department, New York State Psychiatric Institute, 1995.

15 Hamilton M. A rating scale for depression. J Neurol Neurosurg Psychiatry 1960; 23: 56-62.

16 Young RC, Biggs JT, Ziegler VE, Meyer DA. A rating scale for mania: reliability, validity and sensitivity. Br J Psychiatry 1978; 133: 429-35.

17 American Psychiatric Association. Diagnostic and Statistical Manual of Mental Disorders (4th edn) (DSM-IV). APA, 1994.

18 Narisawa-Saito $M$, Nawa $\mathrm{H}$. Differential regulation of hippocampal neurotrophins during aging in rats. J Neurochem 1996; 67: 1124-31.

19 Pillai A, Bruno D, Sarreal AS, Hernando RT, Saint-Louis LA, Nierenberg J, et al. Plasma BDNF levels vary in relation to body weight in females. PLOS One 2012; 7: e39358.

20 Zhang $\mathrm{H}$, Singer B. Recursive Partitioning in the Health Sciences. Springer, 1999.

21 Breiman L, Friedman JH, Olshen RA, Stone CJ. Classification and Regression Trees. Wadsworth, 1984

22 Hosmer DW, Lemeshow S. Applied Logistic Regression (2nd edn). Wiley-Interscience, 2000.

23 World Health Organization. Prevention of Mental Disorders: Effective Interventions and Policy Options. WHO, 2004.

24 Grande I, Fries GR, Kunz M, Kapczinski F. The role of BDNF as a mediator of neuroplasticity in bipolar disorder. Psychiatry Investig 2010; 7: 243-50.

25 Gu L, Xie J, Long J, Chen Q, Chen Q, Pan R, et al. Epidemiology of major depressive disorder in mainland China: a systematic review. PLOS One 2013; 8: e65356.

26 de Oliveira GS, Cereser KM, Fernandes B, Kauer-Sant'anna M, Fries GR, Stertz L, et al. Decreased brain-derived neurotrophic factor in medicated and drug-free bipolar patients. J Psychiatr Res 2009; 43: 1171-4.

27 Fernandes BS, Gama CS, Kauer-Sant'Anna M, Lobato MI, Belmonte-de-Abreu $P$, Kapczinski F. Serum brain-derived neurotrophic factor in bipolar and unipolar depression: a potential adjunctive tool for differential diagnosis. J Psychiatr Res 2009; 43: 1200-4.

28 Machado-Vieira R, Dietrich MO, Leke R, Cereser VH, Zanatto V, Kapczinski F, et al. Decreased plasma brain derived neurotrophic factor levels in unmedicated bipolar patients during manic episode. Biol Psychiatry 2007; 61: $142-4$.
29 Mackin $\mathrm{P}$, Gallagher $\mathrm{P}$, Watson S, Young AH, Ferrier IN. Changes in brainderived neurotrophic factor following treatment with mifepristone in bipolar disorder and schizophrenia. Aust NZ J Psychiatry 2007; 41: 321-6.

30 Sen S, Duman R, Sanacora G. Serum brain-derived neurotrophic factor, depression, and antidepressant medications: meta-analyses and implications. Biol Psychiatry 2008; 64: 527-32.

31 Blugeot A, Rivat C, Bouvier E, Molet J, Mouchard A, Zeau B, et al. Vulnerability to depression: from brain neuroplasticity to identification of biomarkers. J Neurosci 2011; 31: 12889-99.

32 Taylor SM. Electroconvulsive therapy, brain-derived neurotrophic factor, and possible neurorestorative benefit of the clinical application of electroconvulsive therapy. J ECT 2008; 24: 160-5.

33 Arnone D, McKie S, Elliott R, Juhasz G, Thomas EJ, Downey D, et al. State-dependent changes in hippocampal grey matter in depression. Mol Psychiatry 2013; 18: 1265-72.

34 Cubeddu A, Bucci F, Giannini A, Russo M, Daino D, Russo N, et al. Brain-derived neurotrophic factor plasma variation during the different phases of the menstrual cycle in women with premenstrual syndrome. Psychoneuroendocrinology 2011; 36: 523-30.

35 Nakazato M, Tchanturia K, Schmidt U, Campbell IC, Treasure J, Collier DA et al. Brain-derived neurotrophic factor (BDNF) and set-shifting in currently ill and recovered anorexia nervosa (AN) patients. Psychol Med 2009; 39: 1029-35.

36 Li Z, Qi D, Chen J, Zhang C, Yi Z, Yuan C, et al. Venlafaxine inhibits the upregulation of plasma tumor necrosis factor-alpha (TNF- $\alpha$ ) in Chinese patients with major depressive disorder: A prospective longitudinal study. Psychoneuroendocrinology 2013; 38: 107-14.

37 Jacobsen JP, Mork A. The effect of escitalopram, desipramine, electroconvulsive seizures and lithium on brain-derived neurotrophic factor mRNA and protein expression in the rat brain and the correlation to 5-HT and 5-HIAA levels. Brain Res 2004; 1024: 183-92.

38 Omata N, Murata T, Takamatsu S, Maruoka N, Mitsuya H, Yonekura Y, et al. Neuroprotective effect of chronic lithium treatment against hypoxia in specific brain regions with upregulation of cAMP response element binding protein and brain-derived neurotrophic factor but not nerve growth factor: comparison with acute lithium treatment. Bipolar Disord 2008; 10: 360-8.

39 Arnone D, McIntosh AM, Ebmeier KP, Munafò MR, Anderson IM. Magnetic resonance imaging studies in unipolar depression: systematic review and meta-regression analyses. Eur Neuropharmacology 2012; 22: 1-16.

40 Arnone D, Cavanagh J, Gerber D, Lawrie SM, Ebmeier KP, McIntosh AM. Magnetic resonance imaging studies in bipolar disorder and schizophrenia: meta-analysis. Br J Psychiatry 2009; 195: 194-201.

41 Kempton MJ, Geddes JR, Ettinger U, Williams SC, Grasby PM. Meta-analysis, database, and meta-regression of 98 structural imaging studies in bipolar disorder. Arch Gen Psychiatry 2008; 65: 1017-32. 